\title{
Reflexiones sobre la situación actual de la formación de profesionales de odontología en Colombia
}

Reflections on the current dentistry professional education in Colombia

\author{
Reflexões sobre a situação atual da formação de profissionais de \\ Odontologia na Colômbia
}

\section{Cecilia María Martínez Delgado ${ }^{1}$}

\author{
Recibido: 10 de diciembre de 2019 \\ Aprobado: 10 de marzo de 2020 \\ Publicado: 15 de abril de 2020
}

Cómo citar este artículo:

Martínez Delgado CM. Reflexiones sobre la situación actual de la formación de profesionales de odontología en Colombia. Revista Nac. Odontol. (2020); 16(1), 1-8. doi: https://doi.org/10.16925/2357-4607.2020.01.07

Artículo de reflexión. https://doi.org/10.16925/2357-4607.2020.01.07

1 Odontóloga Epidemióloga de la Universidad de Antioquia, Magíster en Dirección Universitaria de la Universidad de los Andes, Profesora jubilada de la Universidad de Antioquia, Profesora de pregrado y posgrado, Facultad de Odontología Universidad CES, Medellín, Colombia.

ORCID: https://orcid.org/0000-0001-8409-7337

Correo electrónico: cmartinezd@ces.edu.co; cmariamar@hotmail.com 


\section{Resumen}

El escenario de la formación de odontólogos en el país es preocupante, no por la calidad de lo que se enseña, sino por la manera como este recurso humano tan importante está pasando por una época de precarización que bien podría contenerse en la medida en que el país reflexione para qué formar profesionales de la salud bucal, si no hay posibilidades de empleo, o si las hay, con un costo muy alto que no se compadece con el retorno a la inversión esperado por los egresados y por sus familias. Este artículo de reflexión quiere generar provocaciones amén de otras que ya se han planteado en el medio nacional, para que entre todos: formadores, decisores de políticas, agremiaciones, los mismos estudiantes, se haga efectiva una planeación de los recursos humanos en salud como lo han reclamado desde hace muchísimas décadas la Organización Mundial de la Salud (OMS), la Organización Panamericana de la Salud (OPS) entre otros.

Palabras clave: educación universitaria, odontología, odontólogos, empleabilidad.

\section{Abstract}

Currently, professional education on dentistry in Colombia is worrying, not because of teaching quality, but because this human resource is going through a precariousness time that could be contained if the country reflects on why educate oral health professionals when there are no employment possibilities or not access to well paid jobs that doesn't allow to recover the investment done. This article aims to provoke that national and education authorities make a accurate planification of health human resources as the WHO and OPS have demanded over the years.

Keywords: university education, dentistry, dentists, employability

\section{Resumo}

O cenário da formação em Odontologia no país é preocupante. Não pela qualidade do que é ensinado, mas sim pela maneira como esse recurso humano tão importante está passando por uma precarização que poderia ser contida à medida que o país refletisse para que formar profissionais de saúde dentária, se não há possibilidades de emprego ou, se há, com um custo muito alto que não compensa o investimento pelos formados e por suas famílias. Este artigo de reflexão pretende gerar provocações além das já propostas no meio nacional para que, entre todos - formadores, decisores de políticas, sindicatos e estudantes -, seja efetivado o planejamen-to dos recursos humanos em saúde como a Organização Mundial da Saúde, a Organização Pan-americana da Saúde, entre outras, têm reivindicado há muitas décadas.

Palavras-chave: educação universitária, odontologia, odontólogos, empregabilidade. 


\section{Introducción}

La formación de estudiantes universitarios reviste un compromiso ético por parte de las instituciones universitarias, llámense instituciones o universidades, así como de los formadores propiamente dichos (docentes). Es un compromiso ético porque se trata de entregar al mercado laboral profesionales competentes, con empleos de calidad y suficiencia para el ejercicio profesional, porque de lo contrario se revela la desarticulación entre la planificación de los recursos humanos en salud en el país, las políticas derivadas de los entes reguladores y el escenario laboral.

Son muchos los programas de pregrado de Odontología con una oferta de egresados muy alta; la mayor preocupación, más allá del empleo, es la empleabilidad, en consecuencia, la calidad de vida de los profesionales de la salud bucal.

El propósito de este escrito es reflexionar sobre la situación actual de la formación y el empleo profesional odontológico, determinados por un sistema de salud en el cual se debe insertar laboralmente y que tiene escasas oportunidades y puestos de trabajo, lo que se debe a la cantidad de diferentes programas que se ofrecen en el país. Esto con el fin de proporcionar orientaciones para el diseño de estrategias tendientes a equilibrar la demanda frente a la oferta, entendiendo que la buena salud bucal de la población colombiana, también es un derecho.

A principios del siglo XVIII, la odontología era ejercida por barberos, curadores o sangradores [1] y su práctica acarreaba un alto costo en cuanto a dolor para quienes solicitaban el servicio. Se ha referido que un "dolor de muelas" es inimaginable, que duele incluso mucho más que un parto normal. Sumado al dolor, entonces, el tratamiento que incluía extracciones dentales, por mucho que doliera, generaba alivio luego del procedimiento, por esto se veían más pacientes desdentados, hecho que también traía consecuencias para la salud general. En esta línea, el primer y segundo estudio de morbilidad oral, realizados en las décadas del 60 y 70, refieren altos índices de pérdida dental expresados en el componente P (perdidos) del índice cop (cariados, obturados y perdidos) [2] [3].

Aunque las exodoncias siguen siendo tratamientos actuales, es claro que conservar los dientes presenta mayores beneficios para la salud a largo plazo; cuando se realizan extracciones dentales se aumentan los indicadores de edentulismo, lo que revela dificultades para acceder de manera oportuna a los servicios de salud. No en vano, la Federación Dental Internacional (FDI) propone "liderar el mundo hacia una salud bucodental óptima, requiere un cambio desde el modelo curativo predominante actual, enfocado a los servicios clínicos individuales del paciente, hacia intervenciones preventivas a nivel poblacional" [4]. Esto quiere decir que los servicios de salud deben considerar la prevención de la enfermedad como uno de sus grandes propósitos, 
mucho más cuando la longevidad y el aumento en la esperanza de vida también están asociados a una buena salud bucal.

La Organización Mundial de la Salud (OMS), en un boletín de prensa en el 2018, se refiere a que "la pérdida grave de dientes y el edentulismo total, fueron algunas de las diez principales causas de años perdidos por discapacidad (APD) en algunos países de altos ingresos" [3]. También la FDı y la oms, en el 2003, establecieron metas específicas como reducir en un porcentaje determinado (que fijarían los países) el número de dientes extraídos por caries en las personas entre 18 y 74 años y el número de dientes extraídos por enfermedad periodontal en el mismo grupo de personas, con especial énfasis por causas como el hábito de fumar, la higiene bucal deficiente, el estrés y las enfermedades sistémicas [5].

El cuarto estudio nacional de salud bucal (ENSAB-IV) indagó por el comportamiento nacional del proceso salud-enfermedad-atención bucal de la población colombiana y mostró una reducción importante en patologías bucodentales más prevalentes, con respecto a los tres estudios realizados con anterioridad en el país. Sin embargo, señala un aumento en la pérdida de dientes como consecuencia de una deficiente atención en salud bucal [6]. Precisamente, las agencias internacionales anteriormente señaladas, indican que "las intervenciones disponibles para prevenir y controlar las enfermedades bucodentales son muchas veces consideradas como un lujo prescindible, más que como un derecho fundamental para todos" [7].

La determinación social de la salud y de la enfermedad [8] tiene varias aristas de comprensión y análisis y, por ello, formar profesionales de salud reviste el mayor compromiso con el aseguramiento de poblaciones e individuos sanos, sin aumentar la cuota de enfermos por cuenta de las políticas de salud y de la prestación de los servicios. Por tanto, podríamos decir, sin temor a equivocarnos, que la misión asumida por las universidades está bastante enredada. Hoy tenemos mayores intervenciones técnicas (especialmente implantes para quienes pueden acceder a ellos) y menos prevención; importante concentración de odontólogos en las ciudades y escasez de profesionales de la salud bucal en los sitios con mayores necesidades de atención; sobreoferta de profesionales (aproximadamente 24000) [9] con precarización en sus condiciones laborales; políticas desacertadas o inexistentes en relación con la salud bucal, de tal manera que los formadores de profesionales en este campo, debemos repensar la tarea con urgencia.

El libro blanco de la odontología editado por el doctor Mariano Sanz en el 2004, hace un balance de la enseñanza de la odontología en el que manifiesta que las facultades de Odontología de la mayoría de los países europeos no están adaptadas a "los tiempos modernos y permanecen ancladas en el pasado más tradicional" [9]. Y esta 
afırmación fue hace 15 años. Hoy en día, muchos artículos periodísticos se refieren a que la mayoría de profesionales no están preparados para atender las necesidades de las empresas, reclamando incluso que la universidad está distanciada de la sociedad porque no consulta sus intereses, sus demandas.

El balance del número de programas universitarios de Odontología en el país es de 33 activos, que se ofrecen en 17 ciudades [11], produciendo en promedio 50 egresados semestralmente, es decir, un número aproximado de 1600 nuevos profesionales cada seis meses, sin contar con los odontólogos ya graduados que ejercen su profesión desde hace varios años. Según el observatorio del talento humano en salud, en el 2015 existían aproximadamente 45069 odontólogos, y si se proyecta un egreso anual de 1500 egresados nuevos, podríamos aproximarnos a casi 54000 para el año 2019, con una razón de un odontólogo por menos de 900 habitantes, una relación "ideal" si se considera la orientación de la oms de un odontólogo por cada 1500 habitantes [12].

Este no es el quid del asunto, es solo una parte de la problemática a considerar: el Ministerio de Salud y Protección Social (MSP) informó que para octubre de 2018 las plazas rurales eran de 568 en todo el país; aunque se sigue llamando servicio social obligatorio (sso), no es obligatorio, depende del número de plazas disponibles, tan claro es que el abecé de seguro social obligatorio expedido por el Minsalud, en el ítem 23 dice textualmente: "se realiza la excepción cuando hay mayor número de profesionales inscritos que plazas disponibles en la profesión correspondiente" [13].

No todos los egresados querrán prestar su servicio social en los 1122 municipios que componen el país, porque no todos están habilitados para la prestación de los servicios de salud bucal (en parte, una excusa por no tener plazas disponibles), por lo que otorga el certificado que exonera de dicho requisito a las cuatro profesiones que se considera deben prestar el año rural como se conoce en el medio. No obstante, tampoco alcanzarían las plazas de acuerdo con el número de egresados semestralmente, por ello, el ejercicio liberal de la profesión sigue teniendo su preponderancia, a pesar de las voces que reclaman que se pasó a la dependencia de las EPS. Pero tanto en el ejercicio independiente como en el dependiente, la realidad constatable es el empobrecimiento o pauperización de los profesionales de la salud bucal.

Otra cara de la situación actual es lo que se denomina "perfil de morbimortalidad" que aún sigue empleándose en el lenguaje epidemiológico y que tiene como propósito mostrar los indicadores de enfermedad y muerte de las poblaciones. El ENSAB-IV [6] reveló que la caries dental no tratada se redujo entre 1965 y 2014 a casi la mitad en las personas con dentición permanente. Sin embargo, la caries dental en los niños de un año, fue un poco menos del 10\% y en los de 3 años llegó a un poco 
más del 40\%. Es evidente que no hay datos de comparación con estudios anteriores, ya que por primera vez se evaluaron estas dos edades. Estos resultados señalan una realidad inconcebible: que en esta primera etapa del desarrollo exista caries dental en pleno siglo XXI, con toda la información que dicen suministrar los servicios de salud y con el desarrollo tecnológico existente; es decir, la primera infancia tiene afectada su salud bucal por patologías prevenibles y estas cifras son aún más comprometedoras, toda vez que hay una política de infancia de orden nacional que habla de desarrollo y atención integral [14].

Ni que decir de la caries cavitada y la caries incipiente, que pueden convertirse en cavidades por ausencia de diagnóstico temprano e intervención oportuna. Es que siguen existiendo barreras al acceso y no solo desde la política misma, sino desde la actitud de los prestadores de salud, transformada en desidia, en profundización de las desigualdades y, por tanto, en inequidad. Al respecto, Velásquez [15] sostiene que "el odontólogo es un operador jurídico del Sistema General de Seguridad Social en Salud colombiano, llamado a tutelar el derecho a la salud en su componente bucal... Estos demuestran conciencia de su responsabilidad social y deóntica... a su vez, exponen las limitaciones arbitrarias a su autonomía profesional por parte de las agencias aseguradoras o prestadoras de servicios de salud (operadores jurídicos dominantes)".

En cuanto a la enfermedad periodontal, entre 1965 y 2014, también se reporta un descenso en su prevalencia (89\% vs 74\% aproximadamente). No obstante, la declinación que, para los más optimistas pudiese significar éxito, no se puede considerar así, pues el dato aportado es de más del 70\%, lo que significaría que podría aumentarse el componente perdido del índice cop, es decir, mayor edentulismo, por cuanto dicho estudio muestra cifras preocupantes: a los 15 años, aproximadamente el 12\% de la población de esta edad presenta pérdida de al menos un diente. El 8\% sufrió pérdida total de los dientes en el grupo de edad de 45 a 59 años y del 34\% entre los 65 y 79 años; de esta manera, las personas mayores tienen afectado seriamente su componente bucal por carecer de dientes, con las consecuencias suficientemente conocidas [6].

Por esto, no es atrevido decir que la tarea de las universidades, en cuanto a la pertinencia que representa formar profesionales de la salud bucal, está extraviada. La pertinencia, en palabras de Malagón [16], es una dimensión compleja con múltiples determinaciones, entendida como "no solo la capacidad de adecuación y adaptación a los contextos sino que pueda ser una institución moderna y crítica, capaz de ejercer influencias, ser participativa y liderar los cambios del entorno, como parte de su objetivo vinculante social, cultural, económico, político y pedagógico" [17]. En este sentido, si la morbilidad bucal no ha logrado un descenso significativo y, ¿por qué no?, un control 
apropiado durante el curso de vida de los habitantes del país, cabe preguntarse ¿en dónde están las facultades de odontología? ¿Y en dónde los egresados? Hay una tarea pendiente deontológica, ética y política.

\section{Conclusiones}

De cada crisis hay resultados positivos. Expresado de otra manera: las crisis siempre son sinónimo de avance, porque nos obligan a pensar de manera creativa qué hacer, cómo replantear los problemas para dar solución de acuerdo con las capacidades y las competencias: los ministerios, que diseñan las políticas; las universidades, que certifican el cumplimiento de requisitos que acredita a los profesionales y, por supuesto, quienes ejercemos la labor de formarlos, los docentes, muchas veces pensando en aportar a su mayoría de edad para el ejercicio crítico, para la transformación y el cambio, para la sostenibilidad, como lo declaran casi todas las misiones institucionales, desconociendo muchas veces, el impacto de su egreso en un mercado laboral adverso.

\section{Referencias}

1. Naranjo C, López H. La Odontología en Colombia: historia, cultura y sociedad. Tomo I. Universidad El Bosque, Bogotá. 2002.

2. República de Colombia, Ministerio de Salud Pública. Investigación Nacional de Morbilidad: Morbilidad Oral. Bogotá, 1971.

3. República de Colombia. Ministerio de Salud. Instituto de Salud. Asociación Colombiana de Facultades de Medicina. Estudio Nacional de Salud y Morbilidad Oral. Bogotá, 1984

4. Federación Dental Internacional (FDI). El desafío de las enfermedades bucodentales. Una llamada a la acción global. Atlas de Salud Bucodental. Myriad Editions Brighton, RU. $2^{\mathrm{a}}$. Ed. 2015. Disponible en: https://www.fdiworlddental.org/sites/default/files/media/documents/ book_spreads_oh2_spanish.pdf

5. Organización Mundial de la Salud (oms). Salud bucodental. Centro de prensa. 24 de septiembre de 2018. Disponible en: https://www.who.int/es/news-room/fact-sheets/detail/ oral-health

6. Ministerio de Salud y Protección Social. IV Estudio Nacional de Salud Bucal (ENSAB-IV). Bogotá. 2015. 
8 Reflexiones sobre la situación actual de la formación de profesionales de odontología en Colombia

7. Hobdell M, Petersen PE, Clarkson J, Johnson N. Global Goals for Oral Health 2020. Int Dent J 2003; 53: 285-288.

8. Breilh J. La determinación social de la salud como herramienta de transformación hacia una nueva salud pública (salud colectiva). Rev. Fac. Nac. Salud Pública 2013; 31(Supl 1): S13-S27.

9. Donatres Giraldo JA, Rojas Amador MA, Jiménez Barbosa WG. Calidad de vida de los odontólogos en Colombia. Revisión exploratoria de la literatura. Univ Odontol. 2017; 36(76): https:// doi.org/10.11144/Javeriana.uo36-76.cvoc

10. Programa de Convergencia Europea de la Agencia Nacional de Evaluación de la Calidad y Acreditación (ANECA). Libro blanco. Título de grado de Odontología. Sanz Alonso M (Coordinador). Madrid, 2004.

11. Ministerio de Educación Nacional de Colombia. Sistema Nacional de Información de la EducaciónSuperior(SNIES).Disponibleen:https://snies.mineducacion.gov.co/consultasnies/ programa\#

12. Organización Mundial de la Salud. Estadísticas sanitarias mundiales. Publicación científica WA 900.1 Suiza. 2012. Disponible en: https://www.who.int/gho/publications/world_health_ statistics/ES_WHS2012_Full.pdf

13. Ministerio de Salud y Protección Social de Colombia. ABECÉ Seguro Social Obligatorio (SSO). Disponible en: https://www.minsalud.gov.co/sites/rid/Lists/BibliotecaDigital/RIDE/VS/TH/ abc-sso.pdf

14. ICBF. Política Nacional de Infanciay Adolescencia 2018-2030. Disponible en: https://www.icbf. gov.co/sites/default/files/politica_nacional_de_infancia_y_adolescencia_2018_-_2030.pdf

15. Velásquez JP. El odontólogo como operador jurídico en el Sistema General de Seguridad Social en Salud colombiano. Tesis de grado. Maestría en Derecho. Universidad de Antioquia [Sin publicar]. Medellín, 2012.

16. Malagón Plata LA. La pertinencia en la educación superior. Elementos para su comprensión. En Revista de la Educación Superior. 2003; XXXII (3), 127. Disponible en internet en: http:// www.anuies.mx/servicios/p_anuies/publicaciones/revsup/127/03.html

17. Castro Aguilar O. Análisis de la dimensión de pertinencia en la educación médica colombiana. Enlace educativo y formativo en salud pública. [Tesis]. Universidad Nacional de Colombia. Facultad de Medicina, Doctorado Interfacultades en Salud Pública. Bogotá D.C. 2016. 